\title{
THE THREE-DIMENSIONAL IMAGING OF ROCK WALL SURFACE WITH THE USE OF COMPUTER-BASED IMAGE PROCESSING AND ANALYSIS
}

\author{
KRZYSZTOF OLESZKO, MARIUSZ MŁYNARCZUK \\ AGH University of Science and Technology; Faculty of Geology, Geophysics and Environmental Protection, \\ Mickiewicza 30, 30-059 Kraków, Poland, e-mail: mlynar@agh.edu.pl
}

\begin{abstract}
The paper presents two methods of digital mapping of rock walls in three dimensional space. The methods have been developed with the use of low-cost devices, which enable image acquisition, and processing methods based on digital image processing and analysis. Both methods are based on so-called shadow profilometry. One of those methods involved mapping the shape of the rock wall on casted mapped surface with analyzed laser lines. The second method was based on the analysis of projected set of bit planes. The planes were projected by computer projector. Both measurement methods have been tested in Miękinia porphyry quarry. The study involved rock wall measurements, which were repeated several times. The part of rock material was removed from the measured wall after each measurement. In order to verify the accuracy of the proposed methods of measurement, volume results obtained and based on digital methods were compared to the volume of removed rock material during measurement. Calculated results were characterized by small deviations which prove to be correct, and are a solid basis for further work on practical application of presented methods.
\end{abstract}

Key words: rock surface, image analysis. 3D imaging, profilometry

\section{INTRODUCTION}

Automation of mining processes, both in underground mines and quarries, requires knowledge of the topography of the excavation, and in particular, the shape of the wall where mining is carried out. It is especially important when the aim is to minimize and, ultimately, to exclude the human participation in the process of mining. Having this in mind, it becomes necessary to develop automated methods for digital mapping of operated surface of the rock wall. Images of rock wall, depending on the prevailing conditions of both the ground and underground, can be taken with the use of various techniques and devices. One of such techniques is presented in work [3]. The authors presented a low-cost method for the rock wall measurement based on a setup involving computer rangefinder controlled with a set of rotary tables. Such method is effective and reproduces the measured surface accurately, unfortunately it is very time consuming. Of course, fast measure apparatus can be used for that kind of measurement, measuring a large number of points on the mapped surface, however such meas- urement devices are expensive [2], [5]. In this study, the authors used two sets of low-cost devices for measurement and mapping the rock surface in three dimensional space. Both of them have good measurement accuracy and in effect the correct representation of the rock wall in three dimensional space. In addition, one of the methods is low time-consuming and the measurement is very simple. The results obtained seem to be correct and show that the methods discussed can be used even in mining conditions.

\section{METHODOLOGY OF MEASUREMENT}

Rock walls, and in particular their external structure, can be measured with the use of various types of equipment - some of the techniques were presented in [4]. Selection of an appropriate measuring device depends mainly on the measured material, the feasibility of measurement (location of the wall to be measured), the dimensions of the measuring device, measuring time and of course the cost of entire meas- 
urement. Measurement methods presented in this article involve low-cost equipment, and the entire setup is not complicated. Due to the fact that the measurements were based mainly on the image analysis of light projected on the measured surface, a prerequisite for their performance was low ambient light, and that is why the measurements were performed in the evening.

In both cases, the setup involved the device responsible for projecting light image under known angle on the rock wall and the camera. The camera was positioned perpendicular to the measured wall. Measuring devices were set in a way that the light images were bigger than the predetermined measurement surface and the camera lens covered the entire surface being measured. In the case described, rectangular area was measured. The surface area was approximately equal to 0.7 square meter. Distance between devices projecting light images, the camera and the measured rock wall was limited to a few meters. Computer projector and a device emitting laser line were used for the purpose of projecting light images. In both cases the same part of the same rock wall was measured.

\subsection{LASER LINES}

The device emitting a laser line was attached to the computer-controlled rotary table, allowing rotation to a specific angle in the horizontal plane. The setup was mounted at a tripod on a customized height.

The measurement started with setting up the device first to emit laser line at an angle equal to 56 degrees in the horizontal plane taking the measured wall into account. The laser line was set in vertical position. The line that was projected on a rock surface formed a curve (when it was observed perpendicular to the rock surface). The shape of this curve depends on the topography of the rock wall. Next, a rotary table was used to change the angle of laser line projection by 0.3 degree. After each angle change a picture of the wall with curved laser line on it was taken. Figure 1 presents example picture of such line projected on a measured wall. A single measurement utilizing laser lines consists of 52 pictures, which contain curved laser line projected on the measured surface.

After the measurement, some rock material was removed from the rock wall measured and the measurement was repeated producing another set of pictures. Therefore, during both measurements 104 pictures were taken. Both before and after the removal of rock material, the set of setup was the same.

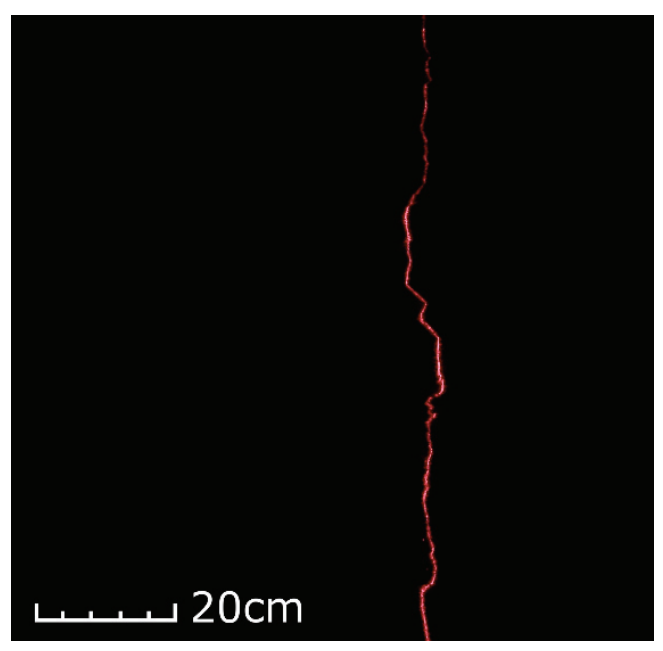

Fig. 1. Example picture of laser line projected on the rock wall being measured

\subsection{BIT PLANES}

The measurements described above require a large number of images. A method for optimizing these measurements seems to be to photograph several measuring lines in a single image. Unfortunately, the complex shape of the curves, and the lack of continuity can prevent the correct separation of the lines in the image. Moreover, multicolored lines, contrary to what it may seem, will not be separated in a sufficient way. This will happen because the rock walls, which by their nature are multicolored, will change the color of the curves displayed.

The solution to this problem is to display on the rock wall the bit planes, and to create the image of multicolored measuring lines in the computer memory. The method is based on the fact that when we display on the bit plane, the points on the rock wall are divided into those for which are illuminated (logical 1) and which are not illuminated (logical 0). For example, if we display 8 bit planes, and the lighting for a given point will vary as: $1,0,0,1,0,0,1,0$ then we receive (for this point) binary value of 10010010 , which corresponds to the decimal value of 146 . If from the obtained image we will select all the points of this value, we obtain the curve whose shape will depend on the topography of the wall. That brings us to the situation written in the previous chapter. It should be noted that by projecting (and photographing) 8 bit planes we can generate 256 easily separable measuring line.

During the measurements, the projector projected bit planes on the rock wall and was placed on a tripod under constant angle equal to 57 degrees in the horizontal plane with respect to the measured wall. The 


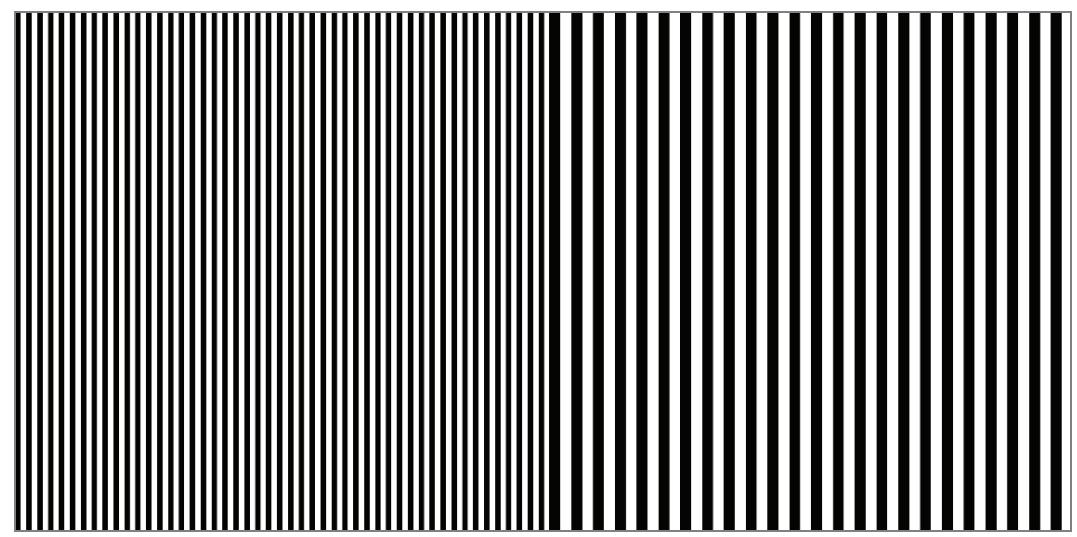

Fig. 2. Bit planes projected on measured surface.

On the left, 16px bit plane. On the right, 32px bit plane

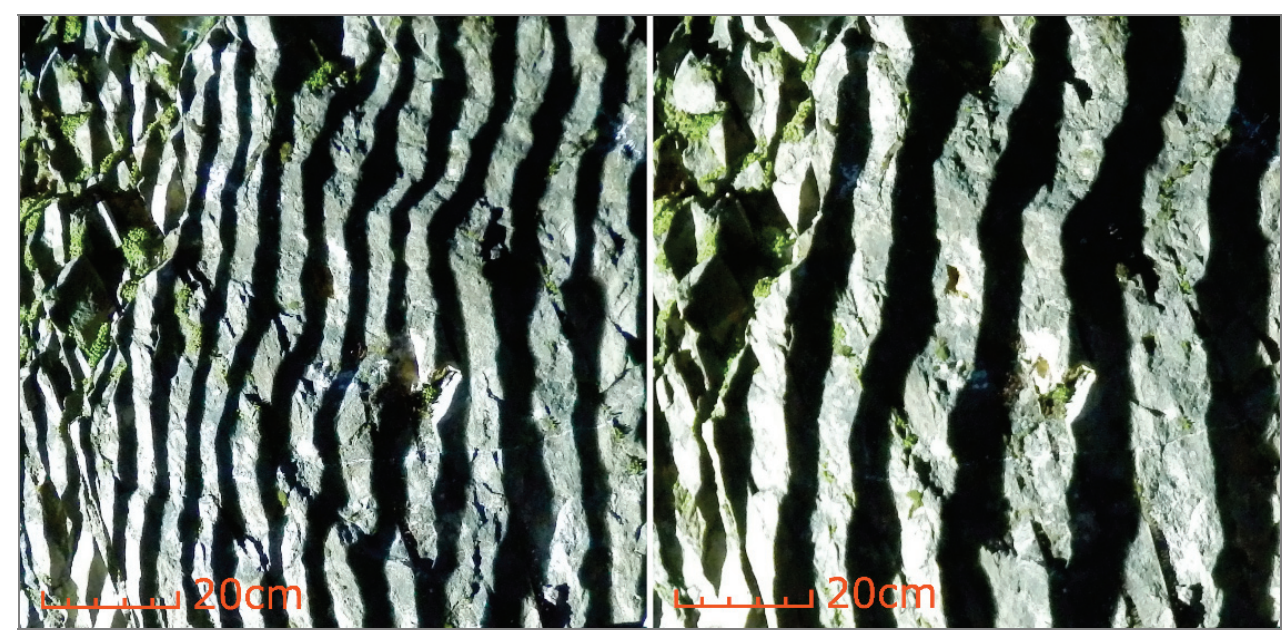

Fig. 3. Curved bit planes on measured rock surface. On the left, 16px bit plane. On the right, 32px bit plane

height at which the projector was located has been suitably chosen - as in the case of the laser device.

During the measurement, the projector remained stationary, while the projected images presenting bit planes were changing. Projected images depicted bit lines (all bit lines of single image produced a bit plane) of different thickness (from 1 to 128 bits) and were arranged vertically. The first of the images presented alternately black and white vertical lines with a thickness of $1 \mathrm{px}$. The other images depicted alternately $2 \mathrm{px}$ line thickness, 4px, 8px, 16px, 32px, 64px and 128px. Figure 2 presents example images used during measurement. After each image change a picture of wall with curved bit planes on it was taken.

Figure 3 presents curved bit planes on measured rock surface.

After conducting the measurement, some rock material was removed from the measured surface and the measurement was repeated producing another set of pictures. A single measurement utilizing bit planes consisted of 8 pictures containing curved bit planes projected on the measured surface. During both measurements, in total 16 pictures were taken. Both before and after removing rock material, the set of setup was the same.

\section{DATA PROCESSING}

The processing of the obtained data, both prior to removal of the rock material and after it, involved the same procedures for each of described methods. The purpose of the processing was surface mapping in the form of three-dimensional computer image. The following description applies to both data sets.

\subsection{LASER LINES}

The acquired images were pre-filtered in order to remove noise. Next, they were binarized and sub- 

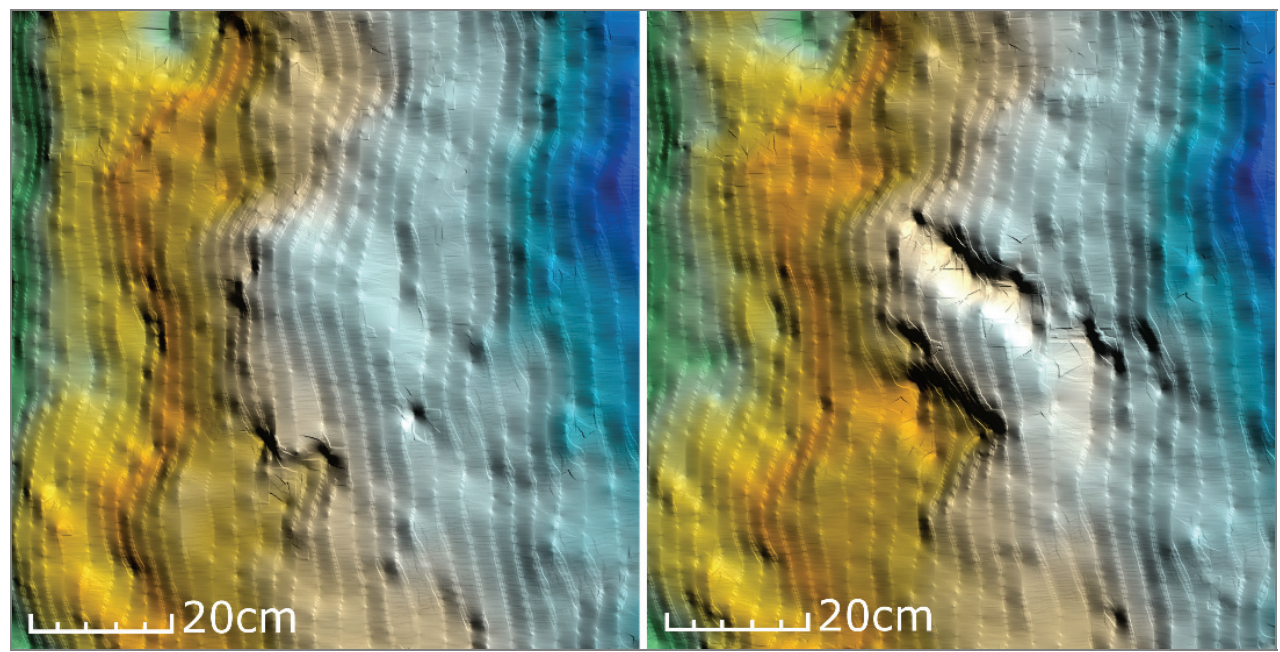

Fig. 4. Measured surfaces before (left) and after (right) removing the rock material

jected to morphological filtering and morphological thinning [6], [7] in order to obtain a single, 1 px thick line representing single laser line. For each of the processed images theoretical line was generated (the line would be created manually and represent the line that would be projected onto a flat surface). The theoretical line together with information about the angle at which the laser line was projected onto measured surface were used to calculate the relative height of the rock wall. The heights were calculated with the use of a triangulation method, inter alia, described and used in [1]. Calculated heights created a grid of points, which described measured surface. In order to get a full three dimensional image of measured rock wall, Kriging method was applied to estimate missing points. Figure 4 presents measured surface before and after removing the rock material.

\subsection{BIT PLANES}

Acquired images representing bit lines were binarized and filtered (morphological opening), so that the alternating black and white lines on the surface of the curved rock wall are clearly visible. Then, after creating 8 bit images, they have been put together in one picture representing the measured surface. Merging all the images was done with the use of the input images used for measurement. Simultaneously there were processed all 8 images. All corresponding bits from resulting images were compared with the same corresponding bits from input bit images. The whole process was repeated in all arrangements of bits until the picture was completed. Then, as in the case of the laser lines, surface with the theoretical data set used to calculate the relative height of the rock wall was gen- erated. The heights were calculated just the same as in the case of the laser line - using the method of triangulation. Calculated heights were used as input to a Kriging method estimating full picture of the measured rock wall.

\section{VERIFICATION OF THE METHODS}

For both data sets, from the laser and projector, after generating, in computer memory, the surface of the rock wall representing measured surface, differences resulting from changes in surface shape were calculated. Figure 5 presents the difference surface calculated from data and obtained before and after removing the rock material.

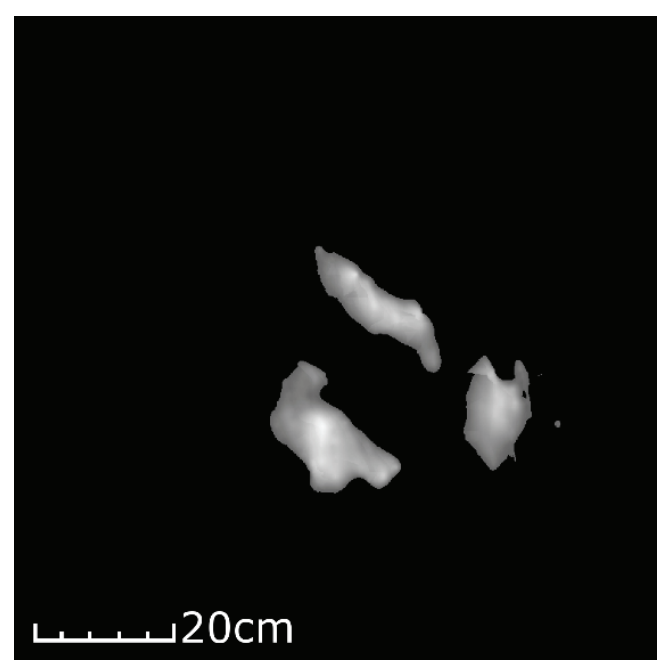

Fig. 5. Difference surface calculated from data obtained before and after removing the rock material 
In order to verify correctness of the digital methods, after removing rock material from the measured wall, the rock material was collected. Then, using a measuring cylinder the volume of collected rock material was measured and compared with the volumes calculated with the use of digital methods. Table 1 presents calculated volume values, and for digital methods, percentage deviations from actual volume value.

Table 1. Calculated volume values and percentage deviations

\begin{tabular}{|c|c|c|c|c|}
\hline Real & \multicolumn{2}{|c|}{ Laser } & \multicolumn{2}{c|}{ Projector } \\
\hline $\begin{array}{c}\text { Volume } \\
{\left[\mathrm{dm}^{3}\right]}\end{array}$ & $\begin{array}{c}\text { Volume } \\
{\left[\mathrm{dm}^{3}\right]}\end{array}$ & $\begin{array}{c}\text { Deviation } \\
{[\%]}\end{array}$ & $\begin{array}{c}\text { Volume } \\
{\left[\mathrm{dm}^{3}\right]}\end{array}$ & $\begin{array}{c}\text { Deviation } \\
{[\%]}\end{array}$ \\
\hline 0.79 & 0.68 & 13.92 & 0.67 & 15.19 \\
\hline
\end{tabular}

\section{DISCUSSION}

Table 1 shows calculated values of the volume employing digital methods, which differ by about $15 \%$ from the actual values. It should be taken into account that during the removal of the rock material, a small part of the rock could not be collected and this affects the actual volume value. Hence the volume values that were digitally calculated, may differ by more than $15 \%$ from the actual one. Taking into account the fact that equipment was inexpensive and the measurements were conducted in-situ, the difference seem to be acceptable. During data processing, images of laser lines were less problematic. They were less noisy and it was relatively easy to obtain a binary image of the curved laser line. The disadvantage of the laser lines method is that it is time consuming. During the measurement, the angle of the laser line was changed by 0.3 degrees - different step can reduce or increase accuracy. With a large surface area going under three dimensional mapping, the process becomes problematic and requires a lot of time. Also, any changes of the angle and the camera shutter synchronization is crucial here. In the case of bit planes, some difficulties were caused by a large amount of noise on images, but after appropriate filtering, positive results were obtained. In the case of the method using bit planes, the measurement was quick and did not require the change of settings of measuring devices. The only thing that has changed was the projected images on the surface of the rock wall. That kind of measurement, regardless of the size of the surface to be measured is not timeconsuming. In addition, depending on the desired accuracy, the number of bit planes can be reduced or increased. Thus, this does not affect the measurement time significantly.

\section{SUMMARY}

The present study describes two methods of three dimensional imaging of rock walls. One method was based on laser lines, and the second one was based on bit planes. In both cases, the images of laser lines and bit planes were filtered and converted for further processing. Based on the resulting data, the rock surface was reproduced in three dimensional space. The volume of rock material removed from the measured rock wall was calculated. In order to verify the obtained results based on digital methods, the volume of removed rock material was measured. Obtained results were compared - digital methods, which helped to calculate the volume produced good results and do not differ significantly from the actual volume value. The method employing the bit planes is noteworthy. It is low-cost, has good results, and first of all the measurement is quick. In addition, presented methods can be used not only for imaging rock walls, but also for any other surface at the macro level. Positive results of this study is a good basis for their continuation on a bigger scale.

\section{ACKNOWLEDGEMENTS}

This work was financed by the AGH - University of Science and Technology, Faculty of Geology, Geophysics and Environmental Protection as a part of statutory project and as the Research Task 15.11.140.338.

\section{REFERENCES}

[1] LeE J.R.J., Smith M.L., SMith L.N., A new approach to the three-dimensional quantification of angularity using image analysis of the size and form of coarse aggregates, Engineering Geology, 2007, Vol. 91, Iss. 2-4, 254-264.

[2] Mah J., Samson C., McKinnon J.D., Thibodeau D., 3D laser imaging for surface roughness analysis, International Journal of Rock Mechanics and Mining Sciences, 2013, Vol. 58, 111-117.

[3] MŁynarczuk M., RatajczaK T., Aksamit J., In-situ measurements of fractures and roughness of rock walls by means of image analysis and laser profilometry, Transactions of the Strata Mechanics Research Institute, 2005, Vol. 7, No. 3-4, 215-229, (in Polish).

[4] Moses C., Robinson D., BARLow J., Methods for measuring rock surface weathering and erosion: A critical review, EarthScience Reviews, 2014, Vol. 135, 141-161. 
[5] Renard F., Voisin C., Marsan D., Schmittbuhl J., High resolution $3 D$ laser scanner measurements of a strike-slip fault quantify its morphological anisotropy at all scales, Geophysical Research Letters, 2006, Vol. 33, DOI: 10.1029/2005GL025038.
[6] SERRA J., Image Analysis and Mathematical Morphology, Academic Press, London 1982.

[7] Serra J., Alternating sequential filters, [in:] J. Serra (Ed.), Image Analysis and Mathematical Morphology, Vol. II, Theoretical Advances, Academic Press, London 1988. 